\title{
Structural insights into a novel family of integral membrane siderophore reductases
}

Inokentijs Josts $^{1 *}$, Katharina Veith ${ }^{1 \#}$, Vincent Normant ${ }^{2,3}$, Isabelle J. Schalk ${ }^{2,3}$, and Henning Tidow $^{1 *}$

1 The Hamburg Advanced Research Center for Bioorganic Chemistry (HARBOR) \& Department of Chemistry, Institute for Biochemistry and Molecular Biology, University of Hamburg, Luruper Chaussee 149, 22761 Hamburg, Germany

${ }^{2}$ CNRS, UMR7242, UMR7242, ESBS, Bld Sébastien Brant, F-67412 Illkirch, Strasbourg, France

3 Université de Strasbourg, UMR7242, ESBS, Bld Sébastien Brant, F-67412 Illkirch, Strasbourg, France

\# present address: European Molecular Biology Laboratory (EMBL), Hamburg Outstation, Hamburg, Germany

* Corresponding authors:

Inokentijs Josts

University of Hamburg, Department of Chemistry, Institute for Biochemistry and Molecular Biology, Luruper Chaussee 149, D-22761 Hamburg, Germany

Tel: +4940428389047

e-mail: josts@chemie.uni-hamburg.de

Henning Tidow

University of Hamburg, Department of Chemistry, Institute for Biochemistry and Molecular Biology, Luruper Chaussee 149, D-22761 Hamburg, Germany

Tel: +4940428388984

e-mail: tidow@chemie.uni-hamburg.de 


\section{Abstract}

Gram-negative bacteria take up the essential ion $\mathrm{Fe}^{3+}$ as ferric-siderophore complexes through their outer membrane using TonB-dependent transporters. However, the subsequent route through the inner membrane differs across many bacterial species and siderophore chemistries and is not understood in detail. Here, we report the crystal structure of the inner membrane protein FoxB (from $P$. aeruginosa) that is involved in Fe-siderophore uptake. The structure revealed a novel fold with two tightly-bound heme molecules. In combination with functional studies these results establish FoxB as an inner membrane reductase involved in the release of iron from ferrioxamine during Fe-siderophore uptake.

Keywords: integral membrane protein, crystal structure, siderophore uptake, reductase, heme 


\section{$\underline{\text { Introduction }}$}

Most microbial communities rely on the bioavailability of iron for their survival. Due to its physico-chemical properties ferric iron exhibits low solubility at physiological $\mathrm{pH}$, precipitating out as ferric oxides and hydroxides. The limited availability of this precious resource has led to severe competition amongst microbes to scavenge this extremely scarce micronutrient from the environment. Most bacterial species, as well as several fungi, secrete numerous small-molecule iron chelators, named siderophores, which bind ferric ion with extremely high affinity and selectivity, at the same time maintaining them in a soluble state. Once such a complex is formed, ferric-siderophores can be taken up into the cells. In Gram-negative bacteria, siderophores are generally taken up by TonB-dependent transporters (TBDTs) present in the bacterial outer membrane $(\mathrm{OM})$. Translocation across the OM relies on the coupling of ferri-siderophorebound TBDTs to the energizing complex in the inner bacterial membrane, comprised of ExbB/ExbD/TonB proteins. Generally, a single TBDT recognizes a subset of siderophores within the same chemical family.

The route of ferric-siderophores across the inner membrane (IM) is less straightforward and differs across many bacterial species and siderophore chemistries. Generally, once inside the periplasmic space, ferric-siderophore complexes are recognized by the dedicated periplasmicbinding proteins for delivery to IM transporters for uptake into the cytoplasm. The most common family of IM transporters involved in the uptake of ferric-siderophores are ATPbinding cassette $(\mathrm{ABC})$ importers. Several $\mathrm{ABC}$ transporters implicated in siderophore transport have been identified and characterized to date (Schalk and Guillon 2013) (Rohrbach, Braun et al. 1995).

In order to assimilate the captured iron into their biological processes, the ferric-siderophore complex must be dissociated. Several mechanisms for iron release from the chelated siderophores have been postulated, which finally all lead to iron reduction, associated either 
with a siderophore hydrolysis (Miethke and Marahiel 2007), chemical modification of the siderophore such as acetylation (Hannauer, Barda et al. 2010) or proton-mediated iron release (Marshall, Stintzi et al. 2009) (Schroder, Johnson et al. 2003) (Miethke and Marahiel 2007). Hydrolysis of siderophores is carried out by dedicated esterases, which fragment the siderophore, lowering the stability of the interaction with iron and facilitating subsequent dissociation steps. To-date, several siderophore hydrolases and esterases have been identified, including Fes, IroD and IroE found in E. coli and Salmonella (Zhu, Valdebenito et al. 2005) (Lin, Fischbach et al. 2005), PfeE from P. aeruginosa (Perraud, Moynie et al. 2018) and Cee from Campylobacter jejuni (Zeng, Mo et al. 2013). Some of these proteins are cytoplasmic, whereas others are localized in the periplasm.

The bacterial cell compartment of iron reduction and release from the siderophore complex also differs based on the microbe and siderophore. Several siderophore reductases have been identified in various bacteria and fungi. For example, E. coli have FhuF, a Fe-S cluster containing protein that targets ferric complexes with coprogen, ferrichrome and ferrioxamine B (Matzanke, Anemuller et al. 2004). Additionally, YqjH, another siderophore reductase found in E. coli, as well as its homologue ViuB from Vibrio cholera, use NADPH-FAD co-factors to reduce iron in ferric-triscatecholates complexes such as enterobactin, aerobactin, vibriobactin and ferric dicitrate (Miethke, Hou et al. 2011). In Gram-positive mycobacteria, IrtAB, a fusion of an $\mathrm{ABC}$ transporter and a reductase is responsible for both the uptake and subsequent reduction of several myco-bacterial siderophores (Arnold, Weber et al. 2020). All these siderophore reductases function in the cytoplasm after the translocation of the ferric-chelate across the bacterial IM.

In stark contrast, certain siderophores rely on iron release within the periplasmic space. For example, in $P$. aeruginosa pyoverdine and citrate siderophores dissociate their iron in the periplasm for subsequent uptake via dedicated Fe transporter, implying the existence of 
siderophore-specific periplasmic reductases (Greenwald, Hoegy et al. 2007) (Ganne, Brillet et al. 2017) (Marshall, Stintzi et al. 2009). Recent studies have identified the four proteins FpvG, FpvH, FpvJ and FpvJ, which co-occur in the pyoverdine uptake operon are responsible for the reduction of iron in the ferric-pyoverdine complex (Ganne, Brillet et al. 2017). The inner membrane FpvG is the reductase, but it needs the two other inner membrane proteins FpvH and FpvI and the periplasmic FpvJ protein for its full activity.

Pseudomonas aeruginosa is a ubiquitous Gram-negative bacterium that has emerged as an opportunistic human pathogen with significant clinical implications. It is one of the leading causes in high mortality rates arising from nosocomial infections. It uses two major siderophores, namely pyoverdine and pyochelin, to obtain iron from the environment. At the same time, it can also utilize foreign siderophores (exosiderophores) as part of its highly adaptable life-style. One such exosiderophore family consists of hydroxamate polydentates named ferrioxamines produced by members of Streptomyces family and several fungi. $P$. aeruginosa, like numerous Gram-negative bacteria, is able to take up ferrioxamines via a dedicated TBDT FoxA in an act of siderophore piracy (Llamas, Sparrius et al. 2006) (Josts, Veith et al. 2019). Interestingly, our recent studies have suggested that ferrioxamine B but not ferrioxamine E (nocardamine) uses an additional, unidentified TBDT for its uptake into $P$. aeruginosa (Normant, Josts et al. 2020). Generally, Gram-negative bacteria use ABC transporters $f h u D B C$ or $h m u U V T$, in a species-specific manner to transport hydroxamate siderophores across the IM (Cuiv, Keogh et al. 2008) (Kingsley, Reissbrodt et al. 1999). However, neither of these uptake systems have been described or characterized in $P$. aeruginosa. Here, we report on the crystal structure of FoxB, which belongs to a large, poorly understood family of inner membrane oxidoreductases associated with siderophore uptake and processing. The protein possesses a novel fold with the transmembrane domain harboring di-hemes indicating a role as inner membrane reductase involved in Fe-siderophore uptake. 


\section{$\underline{\text { Results }}$}

\section{Overall structure of FoxB}

Our previous biochemical and structural studies have implicated the TBDT FoxA in uptake of ferrioxamines $\mathrm{B}$ and $\mathrm{E}$ across the $\mathrm{OM}$ in $P$. aeruginosa. The fox $A$ gene shares its operon with genes encoding for sigma and anti-sigma factors fox $I$ and foxR, respectively, which regulate the operon expression through siderophore-dependent signaling cascades involving the signaling domain of FoxA (Fig. 1a). The operon possesses an additional gene, coding for an uncharacterized IM protein, FoxB, belonging to the conserved family of iron-regulated membrane proteins, termed the PepSY_TM family (COG3182). FoxB and its distant orthologues have been implicated in siderophore uptake but with little functional understanding of their activity (Cuiv, Keogh et al. 2007). Recent studies with a paralogous protein FpvG, found in the pyoverdine operon, have concluded FpvG to be an integral membrane siderophore reductase of ferric pyoverdine (Ganne, Brillet et al. 2017). Therefore, we reasoned that FoxB could also act as a ferrioxamine reductase. To gain insights into the proposed reductase function of FoxB, we have determined the crystal structure of FoxB using X-ray crystallography.

Upon over-expression and purification of FoxB from E. coli membranes it became evident that the protein possesses heme groups with a typical UV-Vis spectrum having a Soret band and a broad Q-band, characteristic of heme-bound proteins. Reducing treatment of FoxB with sodium dithionite resulted in the shift of the Soret peak from $414 \mathrm{~nm}$ to $429 \mathrm{~nm}$, along with a sharp appearance of distinct peaks in the Q-band region (Suppl. Fig. S1a). Oxidation of FoxB with potassium ferricyanide reverted the spectrum to its original state, indicating that FoxB purifies in an oxidized state. Reducing activity of FoxB and subsequent iron dissociation from ferrioxamine B was followed using the colorimetric ferrozine assay, which detects free $\mathrm{Fe}^{2+}$ ions in solution. Purified FoxB had no detectable reductive capacity in any detergents. However, after reconstitution into MSP1D1 nanodiscs, the reductive activity of FoxB could be measured, 
using a range of $\mathrm{Fe}^{3+}$-ferrioxamine $\mathrm{B}$ concentrations (Fig. 1c). Ferrozine forms a complex with $\mathrm{Fe}^{2+}$ resulting in absorbance at $562 \mathrm{~nm}$ and a colour change of solution. The increase in absorbance at $562 \mathrm{~nm}$ could be followed over time in the presence of FoxB, DTT and $\mathrm{Fe}^{3+}-$ ferrioxamine $\mathrm{B}$, indicating that $\mathrm{Fe}^{2+}$ ions were formed by the reduction of the ferric sideophore. We determined the crystal structure of FoxB using single-anomalous dispersion (SAD) in combination with molecular replacement. Initial SAD phases based on the calculated selenium and iron sites (Suppl. Fig. S2) allowed the building of approximately $70 \%$ of the backbone revealing the overall fold of FoxB and placement of heme groups. Some sequence assignment could be made based on the positions of Se atoms, however due to the low number of methionine residues ( 5 total out of 382 residues) and low resolution of the datasets further side chain tracing could not be completed. We then submitted our sequence to the CASP14 (Critical Assessment of Structure Prediction) competition (target T1058) and used the model predicted by the best-performing group (later identified as AlphaFold2 / DeepMind) (Callaway 2020) for molecular replacement. With this model we obtained a clear MR solution, which could then be used for MR-SAD resulting in a good electron density map for entire protein (Suppl. Fig. S3). The structure consists of a four transmembrane (TM) helical bundle capped at the periplasm by two PepSY (peptidase propeptide and YpeB domain / Pfam:PF03413) domains (Fig. 2). The cytoplasmic residues forming a loop between TM2 and TM3 (residues 172 -188) were absent from the electron density and omitted from the final model. The TM bundle wraps around two b-type hemes coordinated non-covalently by highly conserved His residues protruding from the TM domains (Fig. 2 / Suppl. Fig. S4). The iron in both heme groups is in the octahedral coordination state. Both hemes are found at the edges of the lipid bilayers with propionate groups protruding towards the solvent environment. The positioning of the TM bundle in the lipid bilayer was analyzed by the OPM web server (Lomize, Pogozheva et al. 2012) and suggests a tilt angle of $20^{\circ}$ with a hydrophobic thickness of $30.4 \AA$ (Suppl. Fig. S5). TM3 is 
kinked in the middle by a non-conserved stretch of $\mathrm{G}_{201}-\mathrm{G}_{202}$ residues, splitting it into $2 \mathrm{TM}$ helices, TM3a and TM3b. The kink causes TM3a to bend by $66^{\circ}$ perpendicular to the lipid bilayer plane and positions H198 to act as an apical ligand coordinating Fe within the cytoplasmic heme group.

Both propionate groups of the cytoplasmic heme coordinate the conserved residues R10 and K373 through electrostatic or H-bond forces. At the periplasmic side a single heme propionate group is within H-bonding distance of a conserved R342 and a non-conserved Y336 (Fig. 2). The periplasmic PepSY domains form extensive inter-domain contacts through $\beta$-augmentation between the $\beta$-sheets of individual domains as well as additional side-chain H-bond contacts from the adjacent $\alpha$-helices (D260 and S55) (Fig. 3). The buried surface area for the interdomain complex is $479.3 \AA^{2}$. Both PepSY domains enclose a large, solvent-accessible cavity located above the TM domain (Suppl. Fig. S6). This cavity is rich in aromatic amino acids along with a cluster of charged residues in PepSY domain 1. The cluster of negatively charged residues is located on the periplasmic surface of domain 1 (residues 33-117) and a loop from the second PepSY domain (residues 297-300) (Suppl. Fig. S7a).

We could identify two $\mathrm{Zn}$ ions in one of the molecules of FoxB $\left(\mathrm{Zn}_{\mathrm{A} 1}\right.$ and $\left.\mathrm{Zn}_{\mathrm{A} 2}\right)$ as well a single $\mathrm{Zn}$ atom within the second FoxB molecule $\left(\mathrm{Zn}_{\mathrm{B}}\right)$. The crystallization conditions contain $100 \mathrm{mM}$ $\mathrm{ZnCl}_{2}$, which we believe is the source for bound $\mathrm{Zn}$ ions. No crystals of FoxB could be obtained without $\mathrm{Zn}$ supplementation. $\mathrm{Zn}_{\mathrm{A} 1}$ and $\mathrm{Zn}_{\mathrm{A} 2}$ are coordinated by a tetrad of His residues, which form a small tunnel out of the periplasmic cavity. The position of $\mathrm{Zn}_{\mathrm{A} 1}$ and $\mathrm{Zn}_{\mathrm{B}}$ are equivalent but not identical, both ions being in the vicinity of the propionate groups of the periplasmic heme group (Suppl. Fig. S7b).

DALI search suggest a very distant structural similarity of the FoxB TM domains to the $E$. coli superoxide:ubiquinone oxidase ( $\mathrm{pdb}: 5 \mathrm{oc} 0)$ and fumarate reductase (pdb:1e7p) with a root mean square deviation of 5.6 and $5.5 \AA$, respectively (Suppl. Fig. S8a). Both of these are heme- 
containing membrane proteins involved in redox reactions. Analysis of the PepSY domains from FoxB against the available structures of PepSY-containing proteins suggests a highly conserved fold (Suppl. Fig. S8b). The function of these proteins is currently poorly understood with experimental evidence pointing towards inhibition of peptidase activity through proteinprotein interactions. However, these proteins are believed to not possess any TM domains and regulate specific peptidases as stand-alone entities (Yeats, Rawlings et al. 2004).

\section{DFO and NOCA induce foxB and fox $A$ expression}

A Fur box is present upstream of foxI gene (sigma factor) and represses the transcription of fox genes in the presence of iron. In iron deficient conditions, fox $A$ expression is induced (Llamas, Sparrius et al. 2006); this expression is enhanced when the exosiderophores DFO or NOCA are present in the $P$. aeruginosa environment (Llamas, Sparrius et al. 2006) (Normant, Josts et al. 2020). The upregulation of foxA transcription and expression is dependent of the presence of FoxI (Llamas, Sparrius et al. 2006). It has been shown that DFO induces the self-cleavage of the anti-sigma factor FoxR that certainly leads to the release of the sigma factor FoxI (Bastiaansen, Otero-Asman et al. 2015).

To check whether foxB transcription is induced in the presence of DFO or NOCA (as it is the case for foxA), P. aeruginosa PAO1 strain was grown in CAA iron deficient medium with and without $10 \mu \mathrm{M}$ DFO or NOCA. After 8 hours, total RNA was extracted and fox $A$ and fox $B$ transcription were measured by RT-qPCR. As previously described (Normant, Josts et al. 2020), an increase in foxA transcription in the presence of both DFO and NOCA was observed $\left(\log _{2}\right.$ fold change increase of $5.34 \pm 0.45$ for DFO and $5.57 \pm 0.18$ for NOCA) (Fig. 1a). In parallel, fox $B$ transcription was also increased in the presence of DFO $\left(\log _{2}\right.$ fold change increased 3.28 $\pm 0.09)$ and NOCA ( $\log _{2}$ fold change increased $\left.3.23 \pm 0.28\right)$, demonstrating that transcription of both $f o x A$ as well as $f o x B$ genes is regulated by the presence of DFO and NOCA. 


\section{foxB deletion affects iron acquisition by NOCA}

We have previously shown that iron acquisition by NOCA was completely dependent on FoxA outer membrane transporter expression, but iron uptake by DFO was only partially dependent on this transporter (Normant, Josts et al. 2020). To investigate the role of FoxB in these two iron acquisition pathways, ${ }^{55} \mathrm{Fe}$ uptake was monitored in the presence of these two exosiderophores in a PVD and PCH deficient $P$. aeruginosa strain $(\Delta p v d F \Delta p c h A)$ and its corresponding foxA and foxB deletion mutants ( $\triangle p v d F \Delta p c h A \Delta$ fox $A$ and $\triangle p v d F \Delta p c h A \Delta$ foxB)

(Fig. 1b). Strains unable to produce pyoverdine and pyochelin were used to avoid any ${ }^{55} \mathrm{Fe}$ uptake by these two siderophores. These strains were grown in iron restricted CAA medium with $10 \mu \mathrm{M}$ DFO or NOCA to induce the expression of foxA and foxB and the uptake assays were initiated by addition of $500 \mathrm{nM}{ }^{55} \mathrm{Fe}-\mathrm{DFO}$ or ${ }^{55} \mathrm{Fe}-\mathrm{NOCA}$.

Similar rates as previously described for ${ }^{55} \mathrm{Fe}$ uptake in the presence of DFO and NOCA in $\triangle p v d F \triangle p c h A$ cells were observed (Normant, Josts et al. 2020). In the foxA deletion mutant $(\triangle p v d F \Delta p c h A \Delta f o x A)$, transport of ${ }^{55} \mathrm{Fe}-\mathrm{NOCA}$ was totally abolished and partially affected for ${ }^{55} \mathrm{Fe}-\mathrm{DFO}$ as previously described (Fig. 1b): ferri-NOCA is transported across the outer membrane only by FoxA while ferri-DFO is transported by FoxA and at least another TonBdependent transporter (Normant, Josts et al. 2020). foxB deletion had no effect on ${ }^{55} \mathrm{Fe}$ assimilation in the presence of DFO, but partially affected iron uptake by NOCA: $37 \%$ inhibition after 60 minutes and around $26 \%$ after 120 minutes (Fig. 1b). The absence of foxB expression altered iron acquisition by NOCA but not the one by DFO, indicating that FoxB plays a role in iron assimilation by NOCA but is not essential for the DFO iron uptake pathways. FoxB can probably be replaced by another protein that has a redundant function in ferri-NOCA as well as in ferri-DFO uptake pathways. 


\section{Members of COG3182 family are widely distributed across the bacterial kingdom}

Search for FoxB paralogues in the $P$. aeruginosa PAO1 genome revealed the existence of up to five additional operons associated with siderophore or metal ion uptake bearing COG3182 gene members. Each operon also has a dedicated TBDT adjacent to the COG3182 gene. The operons and their associated gene members are outlined in Suppl. Fig. S9. They include the pyochelin operon, putative mycobactin operon and three additional "orphan" operons with no known siderophore substrates (ferrioxamine and pyoverdine operons are excluded).

Members of COG3182 family are widespread across the bacterial kingdom including proteobacteria, firmicutes, bacteroides and cyanobacteria. In Gram-negative bacteria, COG3182 members are often in genomic proximity of a TBDT associated with uptake of siderophores and metal ions as judged by the COGNAT analysis. In several instances we find gene fusions between COG3182 members and sulfite reductases.

\section{Discussion}

Siderophores act to deliver iron, an essential micronutrient, inside the cells of bacteria and fungi. Uptake and translocation of siderophores have received significant scientific attention, yet the precise mechanisms of iron release from these chelated complexes still remain poorly understood. In this study, we report on the structure of FoxB, which belongs to a large, uncharacterized family of integral membrane oxidoreductases that assist in the assimilation of iron from the captured ferric-siderophore complexes in the periplasm through reduction and release of iron from siderophores.

Based on the structure of FoxB, we propose a through-space electron pathway involving the heme molecule on the cytoplasmic side of the TM-region, two conserved Trp residues (W157/W206) located in TM2/3a, the periplasmic heme as well as two $\mathrm{Zn}^{2+}$ ions that may occupy/substitute the $\mathrm{Fe}^{3+}$-siderophore binding site. All residues involved are aligned within a 
distance of $5 \AA$ (Fig. 4). Initial studies into the possible function of FoxB have shown that it was able to substitute the $r h t X$ deletion in uptake of schizokinen, another hydroxamate siderophore in Sinorhizobium meliloti (Cuiv, Clarke et al. 2004). RhtX and its Pseudomonas homologue FptX are members of the major facilitator family of transporters and their roles in siderophore transport are established. We found up to five additional genes (FoxB and FpvG excluded) belonging to the COG3182 family in P. aeruginosa PAO1 strain.

Deletion of $f_{0 x B}$ leads to reduced uptake of iron from NOCA but not ferrioxamine B in $P$. aeruginosa. This result somewhat mirrors our previous study of the TBDT transporter FoxA present in the same operon as FoxB (Josts, Veith et al. 2019). FoxA is able to recognize both ferrioxamine $\mathrm{B}$ and $\mathrm{E}$ with same propensity, however deletion of the fox $A$ gene does not abolish ferrioxamine B uptake (Normant, Josts et al. 2020). Redundancy amongst TBDT towards siderophore uptake is common. The ability of FoxB to assist in the uptake of schizokinen, a hydroxamate siderophore, in $S$. meliloti could also indicate a degree of promiscuity of FoxBlike proteins in P. aeruginosa. An alternative explanation is that ferrioxamine B could be taken up directly into the cytoplasm by an uncharacterized IM transporter. In E. coli, hydroxamate siderophores such as ferrioxamine $\mathrm{B}$ are taken up via the FhuBCD $\mathrm{ABC}$ transporter, which is not present in P. aeruginosa. However, an operon consisting of genes PA2912-2914 encodes for a putative $\mathrm{ABC}$ transporter with distant homology to HmuUVT in P. aeruginosa. This protein complex has been implicated in heme and hydroxamate siderophore uptake in S. meliloti (Cuiv, Keogh et al. 2008). Studies investigating the role of FpvG, a pyoverdine IM reductase in the COG3182 family, have also shown that $f p v G$ mutants reduce ${ }^{55} \mathrm{Fe}$ uptake by pyoverdine in $P$. aeruginosa by approximately $30-50 \%$. It is interesting that FpvG seemingly functions as part of a much larger membrane complex including FpvH, FpvI and FpvJ (Ganne, Brillet et al. 2017), while FoxB seems to function alone. The pyoverdine operon also possesses an ABC transporter $f v p C D E F$, which is implicated in the uptake of iron released from pyoverdine by 
FpvGH (Ganne, Brillet et al. 2017). Since deletion of this transporter reduces uptake by 40$50 \%$, it is possible that iron finds its way into the cells via a different IM transporter. The function of ferrous iron transporter FeoB in the acquisition of iron from ferric-citrate has been demonstrated (Marshall, Stintzi et al. 2009), however it had no role in the transport of iron derived from ferrioxamine B or pyoverdine. Therefore, we cannot rule out the contribution of another IM protein in the translocation of iron ions inside $P$. aeruginosa cells.

\section{$\underline{\text { Materials and Methods }}$}

\section{Chemicals}

Ferrioxamine B (DFO) and the protonophore carbonyl cyanide $m$-chlorophenylhydrazone (CCCP) were purchased from Sigma-Aldrich, ${ }^{55} \mathrm{FeCl}_{3}$ was purchased from Perkin Elmer. NOCA was purified as previously described (Meyer and Abdallah 1980).

\section{Bacterial strains and growth conditions}

The $P$. aeruginosa PAO1 strains used in this study are listed in Table S2. Bacteria were initially grown in LB medium overnight at $30{ }^{\circ} \mathrm{C}$. Next, bacteria were washed and resuspended in irondeficient CAA (casamino acid) medium containing $5 \mathrm{~g} \mathrm{l}^{-1}$ low-iron CAA (Difco), $1.46 \mathrm{~g} \mathrm{l}^{-1}$ $\mathrm{K}_{2} \mathrm{HPO}_{4} 3 \mathrm{H}_{2} \mathrm{O}$ and $0.25 \mathrm{~g} \mathrm{l}^{-1} \mathrm{MgSO}_{4} 7 \mathrm{H}_{2} \mathrm{O}$ and were grown overnight at $30{ }^{\circ} \mathrm{C}$.

\section{Plasmid and strain constructions}

For $\mathrm{pEXG} 2$ fox $B$ construction a 1449 bp insert containing flanking sequences of fox $B$ gene (736 bp upstream and 715 bp downstream foxB gene) was amplified by PCR from genomic DNA of P. aeruginosa PAO1 using the specific primers listed in Table S4 with High-Fidelity DNA polymerase (Thermo-Fisher Scientific). Primers were designed to generate XhoI and HindIII 
restriction sites at 5'end and 3'end of the PCR product. The PCR product was cloned into pEXG2 vector using XhoI and HindIII restrictions sites. Ligation was performed using T4 DNA ligase (Thermo-Fisher Scientific). pEXG2foxB plasmid was transformed in E. coli strain TOP10 (Invitrogen). Mutation in the chromosomal genome of P. aeruginosa $\triangle p v d F \triangle p c h A$ was generated like previously described (Perraud, Cantero et al. 2020) by triparental mating. Mutants were selected and verified by PCR and sequencing.

\section{RNA extractions}

Bacteria were grown overnight in CAA medium. Afterwards, bacteria were pelleted, washed and resuspended at $\mathrm{OD}_{600 \mathrm{~nm}}=0.1$ in fresh CAA medium with or without $10 \mu \mathrm{M}$ NOCA or DFO. Cells were cultivated at $30{ }^{\circ} \mathrm{C}$ for 8 hours. Then, $2.5 \times 10^{8}$ cells were mixed with two volumes of RNAprotect Bacteria Reagent (Qiagen). Samples were lysed in Tris-EDTA (pH 8.0) containing $15 \mathrm{mg} / \mathrm{ml}$ lysozyme (Sigma-Aldrich) for 15 minutes at $25^{\circ} \mathrm{C}$. Lysates were homogenized with QIAshredder kit (Qiagen) and total RNAs were extracted with RNeasy mini kit (Qiagen). After treatment with DNase (RNase-Free DNase Set, Qiagen), RNAs were purified with an RNeasy Mini Elute cleanup kit (Qiagen).

\section{RT-qPCR analysis}

RNA $(1 \mu \mathrm{g})$ was reversed-transcribed with High Capacity RNA-to-cDNA Kit (Applied Biosystems), in accordance with the manufacturer's instructions. The expression of genes was measured in a SpetOne Plus Instrument (Applied Biosystems), with Power Sybr Green PCR Master Mix (Applied Biosystems) and the appropriate primers (Table S3). The $u v r D$ expression was used as an internal control. For a given gene in each strain, the transcript levels were normalized with respect to those for $u v r D$ and were expressed as a base two logarithms of the ratio (fold-change) relative to the reference conditions. 


\section{Iron uptake}

Siderophore- ${ }^{55} \mathrm{Fe}$ complexes were prepared as previously described (Hoegy and Schalk 2014) with a ratio 20:1 for siderophore:iron, in $50 \mathrm{mM}$ Tris- $\mathrm{HCl}$ ( $\mathrm{pH} \mathrm{8.0)}$. After overnight growing in CAA medium, cells were washed and resuspended in fresh CAA medium at $\mathrm{OD}_{600 \mathrm{~nm}}$ of 0.1 in the absence of $10 \mu \mathrm{M}$ NOCA or DFO to induce fox $A$ and fox $B$ expression, and grown overnight at $30{ }^{\circ} \mathrm{C}$. Afterwards, bacteria were washed with $50 \mathrm{mM}$ Tris- $\mathrm{HCl}(\mathrm{pH} 8.0)$, and diluted to an $\mathrm{OD}_{600 \mathrm{~nm}}$ of 1.0. Finally, bacteria were incubated with $500 \mathrm{nM}$ siderophore-- ${ }^{55} \mathrm{Fe}$ at $30^{\circ} \mathrm{C}$. At $\mathrm{t}=0,15,30,45,60$ and 120 minutes, aliquots were removed and cells were harvested by centrifugation and the radioactivity monitored in the bacteria pellet as previously described for iron uptake by pyochelin (Hoegy and Schalk 2014). The assay was carried out as well with bacteria incubated during 15 min with $200 \mu \mathrm{M}$ CCCP in $50 \mathrm{mM}$ Tris- $\mathrm{HCl}(\mathrm{pH} 8.0)\left(\mathrm{OD}_{600 \mathrm{~nm}}\right.$ of 1.0), before addition of $500 \mathrm{nM}$ siderophore- $-{ }^{55} \mathrm{Fe}$.

\section{Protein expression and crystallization}

Full-length $f o x B$ gene from $P$. aeruginosa $P A O 1$ strain was cloned into a pnEK vector with a tobacco etch virus (TEV) cleavable N-terminal His6-tag. Protein was expressed in E. coli $\mathrm{C} 43$ (DE3) cells grown in terrific broth medium. Cells were grown at $37{ }^{\circ} \mathrm{C}$ to an $\mathrm{OD}_{600}$ of 1 and induced with $0.1 \mathrm{mM}$ IPTG followed by overnight growth at $20^{\circ} \mathrm{C}$.

Total membranes were prepared after cell lysis and solubilized in $1 \%(\mathrm{w} / \mathrm{v})$ dodecyl maltopyranoside (DDM) in TBS buffer (20 mM Tris (pH 7.5), $350 \mathrm{mM} \mathrm{NaCl,} 10 \%$ glycerol) for $1 \mathrm{hr}$ at $4{ }^{\circ} \mathrm{C}$. Solubilized membranes were applied onto $\mathrm{Ni}^{2+}$-NTA resin and washed with TBS buffer $+0.03 \%$ DDM and $40 \mathrm{mM}$ imidazole; protein was eluted with $300 \mathrm{mM}$ imidazole and TEV was added overnight (1:5 w/w TEV:protein). Next day, FoxB was reverse purified to remove TEV, the cleaved His 6 -tag and any non-cleaved FoxB protein. Protein was then 
concentrated using a $50 \mathrm{kDa}$ cut-off concentrator and injected onto a Superdex S200 10/300 increase column equilibrated with TBS buffer $+0.2 \%$ decyl maltopyranoside (DM) without glycerol. Purified FoxB protein in TBS buffer $+0.2 \%$ DM was concentrated to $15 \mathrm{mg} / \mathrm{ml}$ and crystallized using sitting-drop vapor diffusion.

\section{Ferrozine reduction assay}

To check the reducing activity of FoxB, the protein was purified as described above using DDM and then incorporated into MSP1D1 nanodiscs in a 1:2:40 ratio (FoxB:MSP1D1:POPC) using previously established protocols. Briefly, proteins and lipids were mixed in the stated ratio, and biobeads $(0.5 \mathrm{mg} / \mathrm{ml})$ were added for 4 hours at $4{ }^{\circ} \mathrm{C}$. Subsequently, assembled protein was removed from biobeads and purified further using a Superdex S200 10/300 column equilibrated in HEPES pH 7, $350 \mathrm{mM} \mathrm{NaCl}$ buffer (buffer B).

For the ferrozine assays, FoxB in nanodiscs (final concentration $3 \mu \mathrm{M}$ ) was mixed with varying concentrations of $\mathrm{Fe}^{3+}$-ferrioxamine $\mathrm{B}$ in buffer $\mathrm{B}$, supplemented with $0.5 \mathrm{mM}$ ferrozine and 30 mM DTT. The mixture was incubated at $37^{\circ} \mathrm{C}$ for $45 \mathrm{~min}$ and absorbance at $562 \mathrm{~nm}$ was then measured. Ferrozine forms a stable magenta-colored complex with absorption peak at $562 \mathrm{~nm}$ with ferrous iron (Stookey 1970). As a negative control, FoxB was omitted from the reaction mixture.

\section{Structure determination}

The structure of FoxB was determined using X-ray crystallography. Diffraction data were collected at $100 \mathrm{~K}$ at the PETRA III/DESY P11, P13 and P14 beamlines. Datasets were processed with XDS (Kabsch 2010) and integrated using AIMLESS (Evans 2011). Due to diffraction anisotropy StarANISO webserver (Tickle, Flensburg et al. 2018) was used to perform anisotropy correction. Heavy atom sites were calculated with SHELX C/D (Sheldrick 
2008). Further density modification was carried out using RESOLVE as part of the PHENIX package (Adams, Afonine et al. 2010). A model generated by the AlphaFold2 group during the CASP14 competition (target T1058) (Callaway 2020) was used for molecular replacement and subsequent MR-SAD. Anomalous difference maps were also used to validate the model. The final model was refined using COOT (Emsley, Lohkamp et al. 2010) and REFMAC (Murshudov, Skubak et al. 2011) using thermal libation and screw-rotation (TLS) and jelly body parameters. All data collection and refinement statistics are summarized in Table S1.

\section{Acknowledgements}

We are grateful to the staff at beamlines P11 (DESY), P13 and P14 (EMBL, Hamburg) and thank members of the Tidow lab for helpful discussions. We acknowledge access to the Sample Preparation and Characterization (SPC) Facility of EMBL. We thank the AlphaFold2 team (DeepMind/Google) for their excellent model (CASP14 target ID: T1058) and Andriy Kryshtafovych (UC Davis) for making CASP14 results available. This research was funded by the excellence cluster 'The Hamburg Centre for Ultrafast Imaging - Structure, Dynamics and Control of Matter at the Atomic Scale' of the Deutsche Forschungsgemeinschaft (DFG EXC 1074).

\section{Author Contributions}

Investigation, I.J., K.V., and V.N.; Writing, I.J., I.J.S. and H.T.; Funding Acquisition \& Supervision, I.J.S and H.T.

\section{Notes}

The authors declare no competing financial interest. 


\section{Footnotes}

The abbreviations used are: ASU, asymmetric unit; CAA, Casamino Acid; CCCP, carbonyl cyanide $m$-chlorophenylhydrazone; DDM, dodecyl maltopyranoside; DFO, Ferrioxamine B; DM, decyl maltopyranoside; CASP14, Critical Assessment of Structure Prediction 14; FoaB, ferrioxamine B; IM, inner membrane; IPTG, isopropyl $\beta$-D-1-thiogalactopyranoside; MR, molecular replacement; Ni-NTA, Ni-nitrilotriacetic acid; NOCA, nocardamine; OM, outer membrane; PCH, pyochelin; PVD, pyoverdine; SAD, single-wavelength anomalous dispersion; SEC, size-exclusion chromatography; TBDT, TonB-dependent transporter; TEV, tobacco etch virus; TM, trans-membrane.

\section{Data Availability}

Structural coordinates and structural factors have been deposited in the RCSB Protein Data Bank under accession number 7ABW (see Table S1, Suppl. Info.). All other relevant data generated during and/or analyzed during the current study are available from the corresponding author on reasonable request.

\section{Significance Statement}

Secretion of siderophores allows most microbes to assimilate ferric ions into their biological processes. Siderophores must be taken up into the cells and chelated iron must be released. Here, we present the structure of an inner membrane siderophore reductase, FoxB, which is involved in the uptake of iron from ferrioxamine siderophores in $P$. aeruginosa. Our structure reveals FoxB to be a di-heme membrane protein, which is able to reduce the iron in chelated ferric siderophore complexes. These results offer insights into the function of this poorly characterized membrane protein family and its role in iron release from bacterial siderophores. 


\section{$\underline{\text { References }}$}

Adams, P. D., P. V. Afonine, G. Bunkoczi, V. B. Chen, I. W. Davis, N. Echols, J. J. Headd, L. W. Hung, G. J. Kapral, R. W. Grosse-Kunstleve, A. J. McCoy, N. W. Moriarty, R. Oeffner, R. J. Read, D. C. Richardson, J. S. Richardson, T. C. Terwilliger and P. H. Zwart (2010). "PHENIX: a comprehensive Python-based system for macromolecular structure solution." Acta Crystallogr D Biol Crystallogr 66(Pt 2): 213-221.

Arnold, F. M., M. S. Weber, I. Gonda, M. J. Gallenito, S. Adenau, P. Egloff, I. Zimmermann, C. A. J. Hutter, L. M. Hurlimann, E. E. Peters, J. Piel, G. Meloni, O. Medalia and M. A. Seeger (2020). "The $\mathrm{ABC}$ exporter IrtAB imports and reduces mycobacterial siderophores." Nature 580(7803): 413-417.

Bastiaansen, K. C., J. R. Otero-Asman, J. Luirink, W. Bitter and M. A. Llamas (2015). "Processing of cell-surface signalling anti-sigma factors prior to signal recognition is a conserved autoproteolytic mechanism that produces two functional domains." Environ Microbiol 17(9): 3263-3277.

Callaway, E. (2020). "'It will change everything': DeepMind's AI makes gigantic leap in solving protein structures." Nature 588(7837): 203-204.

Cuiv, P. O., P. Clarke, D. Lynch and M. O'Connell (2004). "Identification of rhtX and fptX, novel genes encoding proteins that show homology and function in the utilization of the siderophores rhizobactin 1021 by Sinorhizobium meliloti and pyochelin by Pseudomonas aeruginosa, respectively." J Bacteriol 186(10): 2996-3005.

Cuiv, P. O., D. Keogh, P. Clarke and M. O'Connell (2007). "FoxB of Pseudomonas aeruginosa functions in the utilization of the xenosiderophores ferrichrome, ferrioxamine $\mathrm{B}$, and schizokinen: evidence for transport redundancy at the inner membrane." J Bacteriol 189(1): 284-287.

Cuiv, P. O., D. Keogh, P. Clarke and M. O'Connell (2008). "The hmuUV genes of Sinorhizobium meliloti 2011 encode the permease and ATPase components of an ABC transport system for the utilization of both haem and the hydroxamate siderophores, ferrichrome and ferrioxamine B." Mol Microbiol 70(5): 1261-1273.

Emsley, P., B. Lohkamp, W. G. Scott and K. Cowtan (2010). "Features and development of Coot." Acta Crystallogr D Biol Crystallogr 66(Pt 4): 486-501.

Evans, P. R. (2011). "An introduction to data reduction: space-group determination, scaling and intensity statistics." Acta Crystallogr D Biol Crystallogr 67(Pt 4): 282-292.

Ganne, G., K. Brillet, B. Basta, B. Roche, F. Hoegy, V. Gasser and I. J. Schalk (2017). "Iron Release from the Siderophore Pyoverdine in Pseudomonas aeruginosa Involves Three New Actors: FpvC, FpvG, and FpvH." ACS Chem Biol 12(4): 1056-1065.

Greenwald, J., F. Hoegy, M. Nader, L. Journet, G. L. Mislin, P. L. Graumann and I. J. Schalk (2007). "Real time fluorescent resonance energy transfer visualization of ferric pyoverdine uptake in Pseudomonas aeruginosa. A role for ferrous iron." J Biol Chem 282(5): 2987-2995.

Hannauer, M., Y. Barda, G. L. Mislin, A. Shanzer and I. J. Schalk (2010). "The ferrichrome uptake pathway in Pseudomonas aeruginosa involves an iron release mechanism with acylation of the siderophore and recycling of the modified desferrichrome." J Bacteriol 192(5): 12121220 .

Hoegy, F. and I. J. Schalk (2014). "Monitoring iron uptake by siderophores." Methods Mol Biol 1149: $337-346$. 
Josts, I., K. Veith and H. Tidow (2019). "Ternary structure of the outer membrane transporter FoxA with resolved signalling domain provides insights into TonB-mediated siderophore uptake." Elife 8.

Kabsch, W. (2010). "Xds." Acta Crystallogr D Biol Crystallogr 66(Pt 2): 125-132.

Kingsley, R. A., R. Reissbrodt, W. Rabsch, J. M. Ketley, R. M. Tsolis, P. Everest, G. Dougan, A. J. Baumler, M. Roberts and P. H. Williams (1999). "Ferrioxamine-mediated Iron(III) utilization by Salmonella enterica." Appl Environ Microbiol 65(4): 1610-1618.

Lin, H., M. A. Fischbach, D. R. Liu and C. T. Walsh (2005). "In vitro characterization of salmochelin and enterobactin trilactone hydrolases IroD, IroE, and Fes." J Am Chem Soc 127(31): 11075-11084.

Llamas, M. A., M. Sparrius, R. Kloet, C. R. Jimenez, C. Vandenbroucke-Grauls and W. Bitter (2006). "The heterologous siderophores ferrioxamine B and ferrichrome activate signaling pathways in Pseudomonas aeruginosa." J Bacteriol 188(5): 1882-1891.

Lomize, M. A., I. D. Pogozheva, H. Joo, H. I. Mosberg and A. L. Lomize (2012). "OPM database and PPM web server: resources for positioning of proteins in membranes." Nucleic Acids Res 40(Database issue): D370-376.

Marshall, B., A. Stintzi, C. Gilmour, J. M. Meyer and K. Poole (2009). "Citrate-mediated iron uptake in Pseudomonas aeruginosa: involvement of the citrate-inducible FecA receptor and the FeoB ferrous iron transporter." Microbiology (Reading) 155(Pt 1): 305-315.

Matzanke, B. F., S. Anemuller, V. Schunemann, A. X. Trautwein and K. Hantke (2004). "FhuF, part of a siderophore-reductase system." Biochemistry 43(5): 1386-1392.

Meyer, J.-M. and M. A. Abdallah (1980). "The Siderochromes of Non-fluorescent Pseudomonads: Production of Nocardamine by Pseudomonas stutzeri." Microbiology 118: 125129.

Miethke, M., J. Hou and M. A. Marahiel (2011). "The siderophore-interacting protein YqjH acts as a ferric reductase in different iron assimilation pathways of Escherichia coli." Biochemistry 50(50): 10951-10964.

Miethke, M. and M. A. Marahiel (2007). "Siderophore-based iron acquisition and pathogen control." Microbiol Mol Biol Rev 71(3): 413-451.

Murshudov, G. N., P. Skubak, A. A. Lebedev, N. S. Pannu, R. A. Steiner, R. A. Nicholls, M. D. Winn, F. Long and A. A. Vagin (2011). "REFMAC5 for the refinement of macromolecular crystal structures." Acta Crystallogr D Biol Crystallogr 67(Pt 4): 355-367.

Normant, V., I. Josts, L. Kuhn, Q. Perraud, S. Fritsch, P. Hammann, G. L. A. Mislin, H. Tidow and I. J. Schalk (2020). "Nocardamine-Dependent Iron Uptake in Pseudomonas aeruginosa: Exclusive Involvement of the FoxA Outer Membrane Transporter." ACS Chem Biol 15(10): 2741-2751.

Perraud, Q., P. Cantero, B. Roche, V. Gasser, V. P. Normant, L. Kuhn, P. Hammann, G. L. A. Mislin, L. Ehret-Sabatier and I. J. Schalk (2020). "Phenotypic Adaption of Pseudomonas aeruginosa by Hacking Siderophores Produced by Other Microorganisms." Mol Cell Proteomics 19(4): 589-607.

Perraud, Q., L. Moynie, V. Gasser, M. Munier, J. Godet, F. Hoegy, Y. Mely, G. L. A. Mislin, J. H. Naismith and I. J. Schalk (2018). "A Key Role for the Periplasmic PfeE Esterase in Iron Acquisition via the Siderophore Enterobactin in Pseudomonas aeruginosa." ACS Chem Biol 13(9): 2603-2614.

Rohrbach, M. R., V. Braun and W. Koster (1995). "Ferrichrome transport in Escherichia coli K-12: altered substrate specificity of mutated periplasmic FhuD and interaction of FhuD with the integral membrane protein FhuB." J Bacteriol 177(24): 7186-7193. 
Schalk, I. J. and L. Guillon (2013). "Fate of ferrisiderophores after import across bacterial outer membranes: different iron release strategies are observed in the cytoplasm or periplasm depending on the siderophore pathways." Amino Acids 44(5): 1267-1277.

Schroder, I., E. Johnson and S. de Vries (2003). "Microbial ferric iron reductases." FEMS Microbiol Rev 27(2-3): 427-447.

Sheldrick, G. M. (2008). "A short history of SHELX." Acta Crystallogr A 64(Pt 1): 112-122.

Stookey, L. L. (1970). "Ferrozine - A new spectrophotometric reagent for iron." Anal. Chem. 42(7): 779-781.

Tickle, I. J., C. Flensburg, P. Keller, W. Paciorek, A. Sharff, C. Vonrhein and G. Bricogne (2018). "STARANISO (http://staraniso.globalphasing.org/cgi-bin/staraniso.cgi). Cambridge, United Kingdom: Global Phasing Ltd. ."

Yeats, C., N. D. Rawlings and A. Bateman (2004). "The PepSY domain: a regulator of peptidase activity in the microbial environment?" Trends Biochem Sci 29(4): 169-172.

Zeng, X., Y. Mo, F. Xu and J. Lin (2013). "Identification and characterization of a periplasmic trilactone esterase, Cee, revealed unique features of ferric enterobactin acquisition in Campylobacter." Mol Microbiol 87(3): 594-608.

Zhu, M., M. Valdebenito, G. Winkelmann and K. Hantke (2005). "Functions of the siderophore esterases IroD and IroE in iron-salmochelin utilization." Microbiology (Reading) 151(Pt 7): 2363-2372. 


\section{Figures and figure legends}

\section{Figure 1}

A

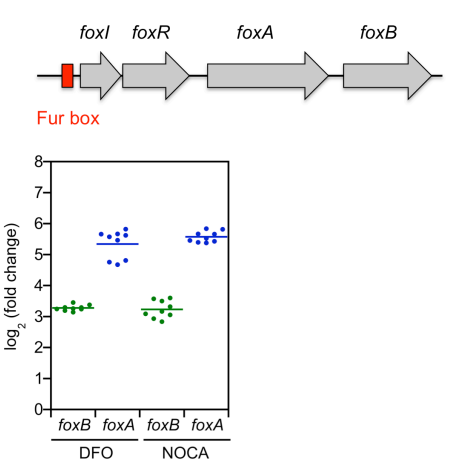

B

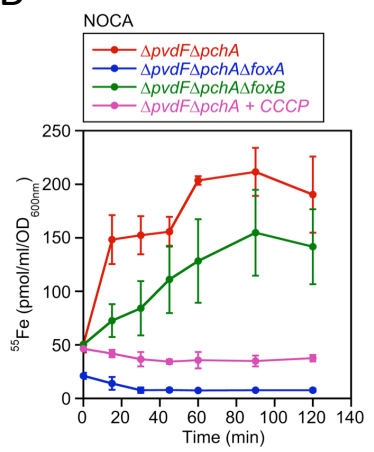

C

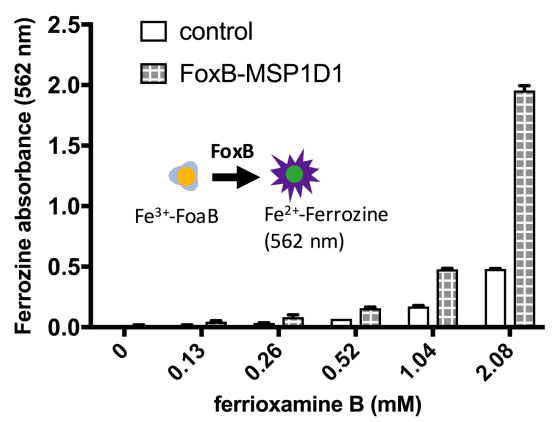

Functional characterization of $\boldsymbol{f o x B}$. A) Expression of $f \circ x B$ and $f \circ x A$ in presence of DFO or NOCA in minimal medium. Top panel: Organization of fox genes in P. aeruginosa genome. fox $A$ encode for TBDT transporter localize at the outer membrane, foxI encode for sigma factor, foxR encode for anti-sigma factor localize at the inner membrane and fox $B$ encode for protein localize at the inner membrane. A fur box is present upstream foxI gene. Bottom panel: PAO1 WT was grown in CAA medium overnight and, then resuspended in fresh CAA medium at $\mathrm{OD}_{600 \mathrm{~nm}}=0.1$ and treated or not with $10 \mu \mathrm{M}$ DFO or $10 \mu \mathrm{M}$ NOCA for 8 hours. Total RNA was extracted and retro-transcribed in cDNA. Expression of genes was normalized to the $u v r D$ reference gene in each condition. The expression of $f_{0 x A}$ and $f_{0 x B}$ was measured in DFO or NOCA condition and related to untreated condition. Results were represented in $\log _{2}$ fold change expression. Graphical represent the result of three biological experiments each performed in triplicate. The bars represent the means. B) Transport of ${ }^{55} \mathrm{Fe}-\mathrm{NOCA}$ in PVD and $\mathrm{PCH}$ biosynthesis deficient cells expressing or not foxA or foxB. PAO1 $\triangle p v d F \Delta p c h A$, $\triangle p v d F \Delta p c h A \Delta$ foxA and $\triangle p v d F \Delta p c h A \Delta$ fox $B$ were grown in CAA medium with $10 \mu \mathrm{M}$ NOCA to induce fox $A$ and $f \circ x B$ expression overnight at $30^{\circ} \mathrm{C}$. Then, cells were washed with $50 \mathrm{mM}$ Tris- $\mathrm{HCl}(\mathrm{pH} 8.0)$. The transport assay was started by the addition of $500 \mathrm{nM}{ }^{55} \mathrm{Fe}-\mathrm{NOCA}$ to $\triangle p v d F \triangle p c h A$ (red curve), $\triangle p v d F \triangle p c h A \Delta f o x A$ (blue curve) and $\triangle p v d F \Delta p c h A \Delta f o x B$ (green curve). The $\triangle p v d F \triangle p c h A$ strain was also treated with $200 \mu \mathrm{M} \mathrm{CCCP}$ protonophore (pink curve) as a control in presence of ${ }^{55} \mathrm{Fe}-\mathrm{NOCA}$. At each indicated point (0 to 120 minutes), samples were centrifuged, and the radioactivity retained in the cell pellets were monitored. Data represent the mean of ${ }^{55} \mathrm{Fe}$ concentration in $\mathrm{pmol} / \mathrm{ml} / \mathrm{OD}_{600 \mathrm{~nm}} \pm \mathrm{SD}$ of three independent experiments. C) Ferrioxamine $\mathrm{B}$ reduction assay using ferrozine absorption to monitor $\mathrm{Fe}^{2+}$ release from the siderophore. Absorbance was measured at $562 \mathrm{~nm}$ for a range of ferrioxamine 
concentrations and FoxB in MSP1D1 $(3 \mu \mathrm{M})$ and $15 \mathrm{mM}$ DTT. Plots represent an average of three independent assays with standard error shown.

\section{Figure 2}
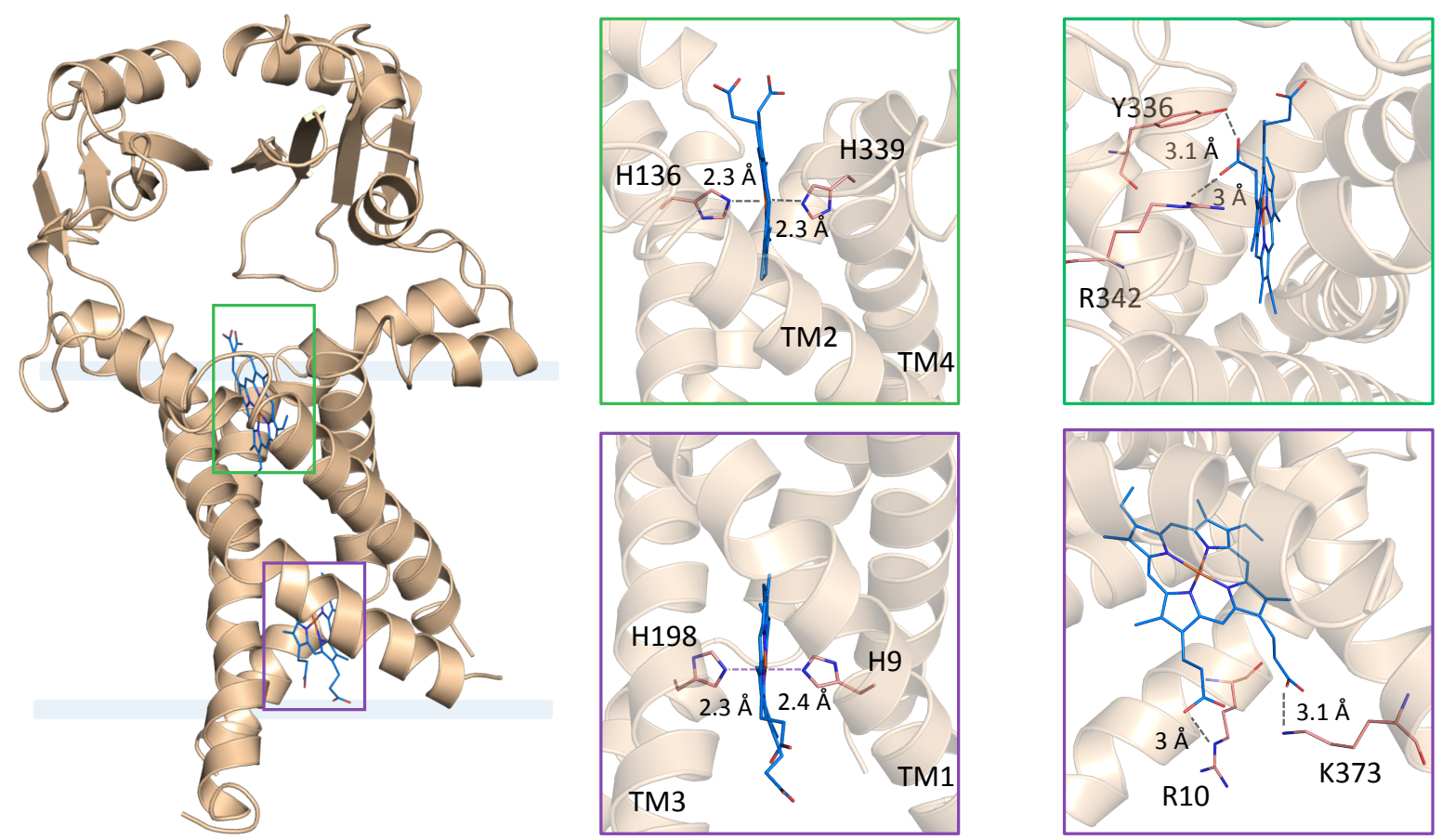

Overall structure of FoxB. A) FoxB structure reveals a 4-helix TM-fold topped with periplasmic PepSY domains. The membrane-spanning region is indicated and both heme molecules are boxed. B) Di-heme coordination by the TM domain of FoxB. All four transmembrane helices contribute to heme binding. Both hemes are found at the edges of the lipid bilayers with propionate groups protruding towards the solvent environment. Coordinating residues towards the $\mathrm{Fe}$ atoms or propionate groups are indicated for both hemes in two orientations. 


\section{Figure 3}
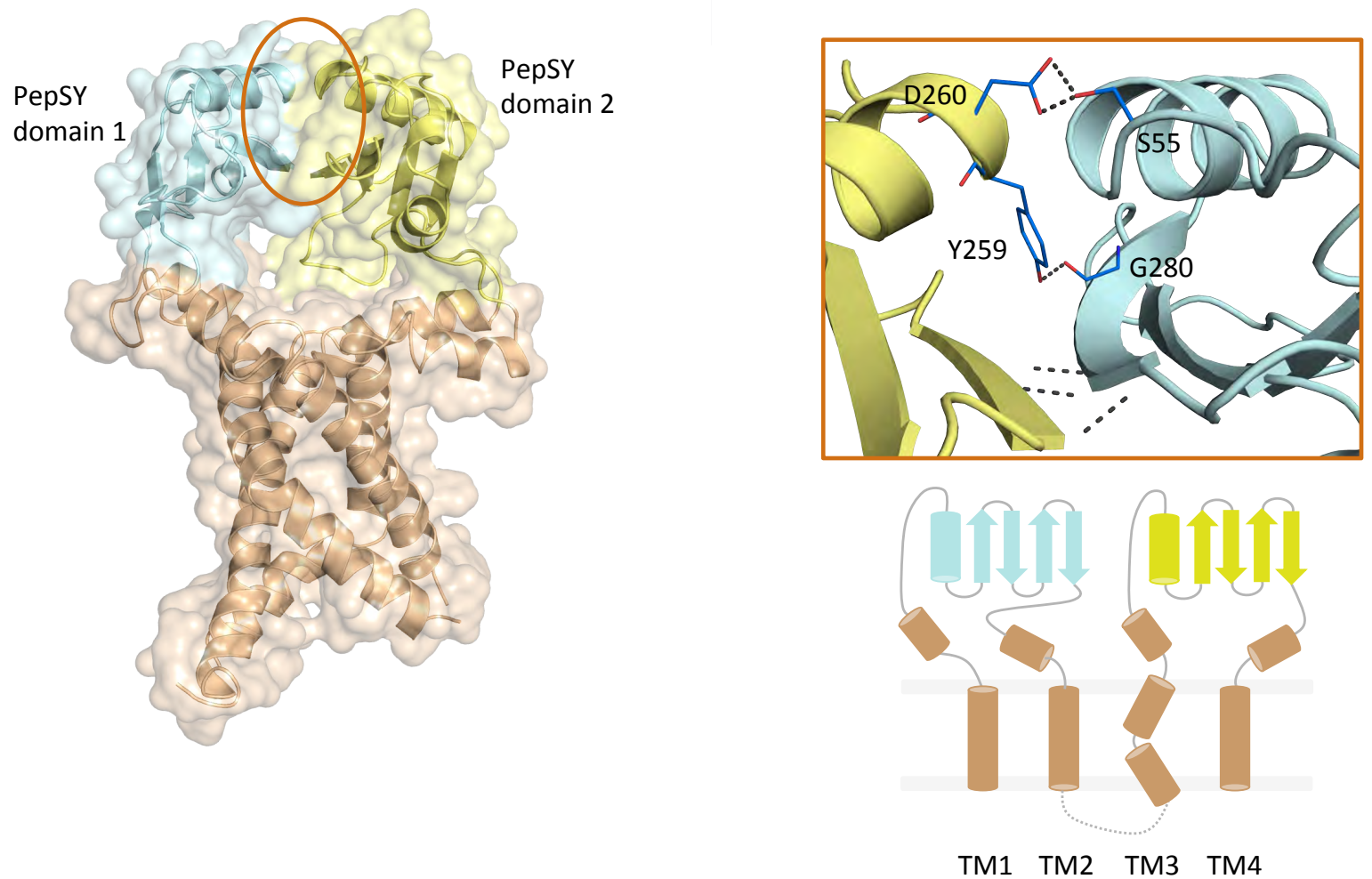

Periplasmic PepSY domains within FoxB. A) Two PepSY domains (residues 46-118 and 239-325, respectively) form the periplasmic part of the protein and interact via several hydrogen bonds (shown in B). Overall secondary structure of FoxB is also shown. 


\section{Figure 4}
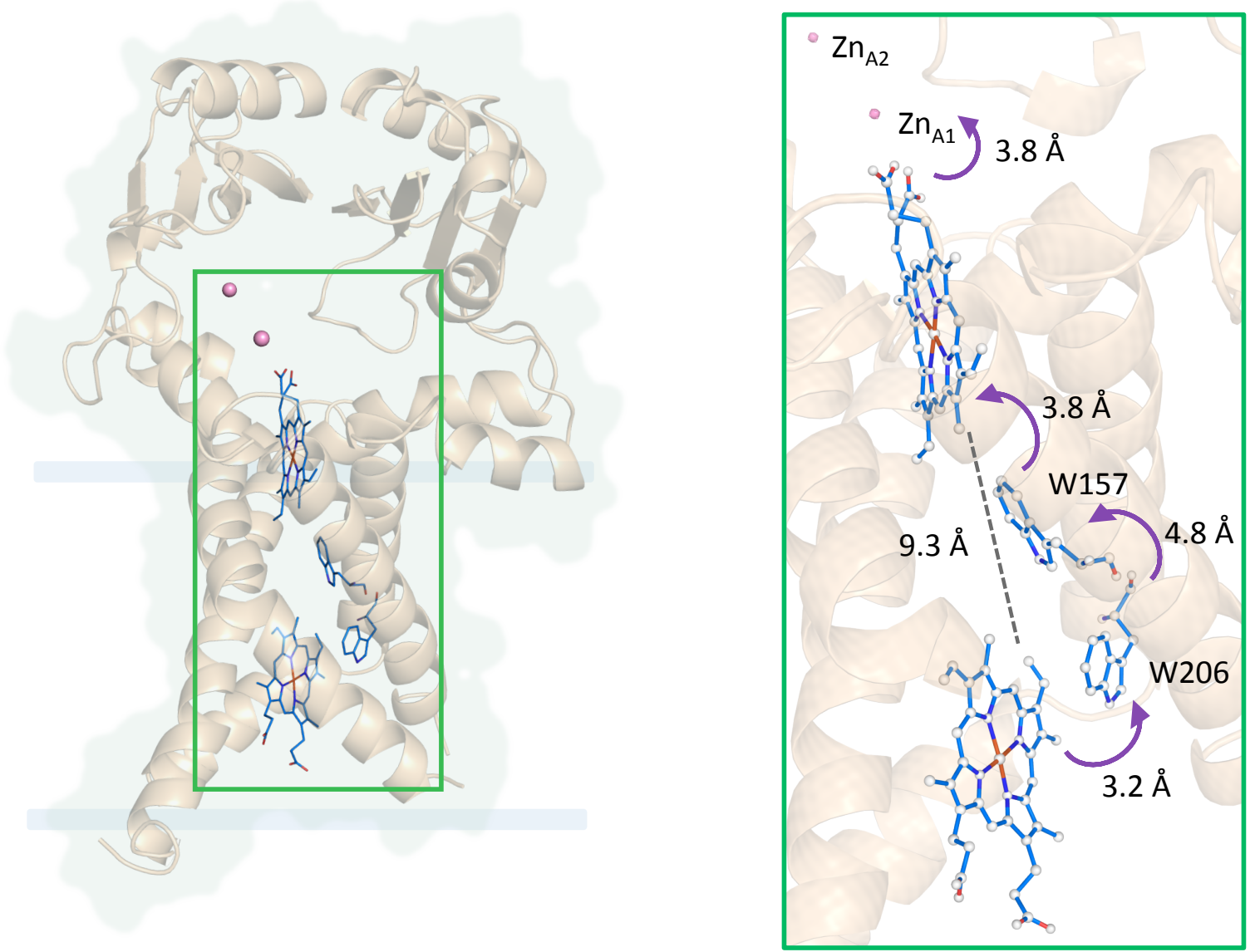

Potential electron pathway in FoxB reductase. The proposed through-space electron pathway comprises the heme molecule on the cytoplasmic side of the TM-region, two highly conserved Trp residues (W157/W206) located in TM2/3a, the periplasmic heme as well as two $\mathrm{Zn}^{2+}$ ions that may occupy/substitute the $\mathrm{Fe}^{3+}$-siderophore binding site. All residues involved are aligned within a distance of $5 \AA$. 